\title{
Effects of anxiety and depression symptoms on oxidative stress in patients with alopecia areata
}

\author{
Gokhan Cakircaํ, Vildan Manav², Hakim Celik ${ }^{3}$, Gülben Saracoglư ${ }^{4}$ Esra Nur Yetkin ${ }^{1}$ \\ ${ }^{1}$ Department of Biochemistry, Sanliurfa Mehmet Akif Inan Training and Research Hospital, Sanliurfa, Turkey \\ ${ }^{2}$ Department of Dermatology, Istanbul Training and Research Hospital, Istanbul, Turkey \\ ${ }^{3}$ Department of Physiology, Faculty of Medicine, Harran University, Sanlıurfa, Turkey \\ ${ }^{4}$ Department of Psychiatry, Sanliurfa Training and Research Hospital, Sanliurfa, Turkey
}

Adv Dermatol Allergol 2020; XXXVII (3): 412-416 DOI: https://doi.org/10.5114/ada.2019.83879

\begin{abstract}
Introduction: Increased oxidative stress (OXS) and a high prevalence of psychiatric disorders are seen in alopecia areata (AA). However, OXS and psychiatric disorders have been studied separately in AA patients.

Aim: To determine the effects of anxiety and depression symptoms on OXS in AA patients.

Material and methods: The anxiety and depression levels of 33 AA patients and 33 normal controls (NC) were determined using the Hospital Anxiety and Depression Scale. The oxidative stress index (OSI) was calculated by measuring serum total antioxidant status (TAS) and total oxidant status (TOS) levels in AA patients and NC.

Results: The AA patients had higher anxiety and depression scores than NC ( $p<0.001$ for both). Total oxidant status $(p=0.002)$ and OSI $(p<0.001)$ values were higher, and TAS $(p<0.001)$ levels were lower, in patients with AA compared to NC. However, patients' anxiety and depression scores were not correlated with the TAS, TOS, or OSI values $(p>0.05)$. There was no significant difference in TAS, TOS, or OSI values between patients with high and low anxiety or depression scores $(p>0.05)$.

Conclusions: These results show that OXS, anxiety, and depression scores were higher in patients with AA compared to NC. However, anxiety and depression scores were not associated with OXS in AA patients. More extensive studies should be performed to investigate the relationship between psychological status and OXS in patients with AA.
\end{abstract}

Key words: alopecia areata, oxidative stress, anxiety, depression.

\section{Introduction}

Alopecia areata (AA) is an autoimmune and inflammatory disease characterized by recurrent hair loss without scarring on the scalp and/or body. AA, which can occur in all age groups, commonly affects men and women equally [1]. Although the etiopathogenesis of AA has not been fully elucidated, genetic predisposition, T-cellmediated immunological reactions, and environmental factors such as hormones, diet, vaccinations, and stress are considered to play a role in the development of this disease [2, 3].

The skin, which protects the body from external factors, is continuously exposed to endogenous and environmental pro-oxidant agents, leading to the overproduction of reactive oxygen species (ROS). Reactive oxygen species are thought to activate proliferative and cell signaling pathways, which can in turn alter apoptotic pathways that may play a pivotal role in the pathogenesis of many dermatological diseases [4]. The tissue damage caused by increased ROS is prevented by enzymatic and non-enzymatic antioxidant agents [5]. Oxidative stress (OXS) caused by deterioration of the balance between ROS and the antioxidant defense system may play a role in the pathogenesis of AA [6, 7].

Hair loss, especially on the scalp, has a negative effect on the esthetic appearance and self-esteem of individuals. Therefore, patients suffering from AA may be prone to various psychiatric illnesses, such as anxiety and depression [8]. Some researchers also emphasized that stressful life events may play an important role in the onset or progression of the disease $[9,10]$. Studies have shown that the prevalence of psychiatric disorders such as depression and anxiety is higher in AA patients than in healthy controls [11, 12].

\footnotetext{
Address for correspondence: Gokhan Cakirca MD, Biochemistry Department, Sanliurfa Mehmet Akif Inan Training and Research Hospital, Sanliurfa, Turkey, phone: +90 414318 6000, fax: +90 414318 6707, e-mail: cakirca.gokhan@gmail.com Received: 29.01.2019, accepted: 20.02.2019. 
Although psychiatric disorders and OXS status have been investigated independently in AA patients, the relationship between psychiatric disorders and OXS was not evaluated in these patients.

\section{Aim}

In this study, we aimed to determine the effects of depressive and anxiety symptoms on OXS in patients with AA.

\section{Material and methods \\ Participants}

Thirty-three AA patients and 33 normal controls (NC) were included in this study. The AA patients were enrolled from the dermatology outpatient clinic of Sanliurfa Mehmet Akif Inan Training and Research Hospital (Turkey), between March and December 2017. The demographic characteristics of the patients (age and gender, disease duration, recurrences, family history, presence of stress, and pattern of AA) were recorded. The Severity of Alopecia Tool score for AA was used to determine the degree of hair loss based on the percentage of scalp surface area involved. Exclusion criteria were concomitant systemic diseases, acute/chronic infection, use of any treatment including antioxidant vitamins and systemic or topical medications over the last 3 months, smoking, and alcohol intake. Ethical approval for this study was obtained from the local ethics committee.

\section{Measurement of oxidative stress parameters}

Venous blood was obtained from AA patients and NC to measure the total antioxidant status (TAS) and total oxidant status (TOS) after 8-12 h of fasting. The collected blood samples were centrifuged and the serum was stored in Eppendorf tubes at $-80^{\circ} \mathrm{C}$ until needed. Serum

Table 1. Demographic characteristics in patients with alopecia areata

\begin{tabular}{lc}
\hline Parameter & Value \\
\hline Gender (male) & $25(75.8 \%)$ \\
\hline Disease duration [months] & $3(1-48)$ \\
\hline Family history & $6(18.2 \%)$ \\
\hline Recurrence & $7(21.2 \%)$ \\
\hline Stress & $24(72.7 \%)$ \\
\hline Pattern: & \\
\hline Patch & $31(93.9 \%)$ \\
\hline Patch + ophiasis & $2(6.1 \%)$ \\
\hline SALT score: & $27(81.8 \%)$ \\
\hline S1 & $5(15.2 \%)$ \\
\hline S2 & $1(3 \%)$ \\
\hline S3
\end{tabular}

levels of TOS and TAS were measured using the fully automated colorimetric method described by Erel [13, 14]. After determination of TOS and TAS levels, oxidative stress index (OSI) was calculated. The ratio of TOS to TAS yields the OSI, an indicator reflecting the severity of OXS.

\section{Hospital Anxiety and Depression Scale (HADS)}

The HADS, which is used to evaluate patients' anxiety (HADS-A; 7 items) and depression (HADS-D; 7 items) scores, consists of 14 items. HADS subscale scores range from 0 to 21 [15]. In a study on the validity and reliability of the Turkish version of HADS by Aydemir et al., cut-off scores were determined as 10 for the HADS-A subscale and 7 for the HADS-D subscale [16]. In this study, we used the Turkish version of the scale to evaluate the anxiety and depression symptoms of patients with AA.

\section{Statistical analysis}

Statistical calculations were carried out using IBM SPSS Statistics 22 (IBM Corp., Armonk, NY). A p-value $<0.05$ was considered to indicate statistical significance. The normality of the variables was assessed by the Kolmogorov-Smirnov test. Differences between the groups were determined using Student's t-test, the Mann-Whitney $U$-test or the $\chi^{2}$ test. The relationship between HADS subscale scores and OXS markers in AA patients was evaluated by Pearson's correlation test.

\section{Results}

This study included 33 patients with AA (25 males/ 8 females) with a mean age of $26.33 \pm 6.08$ years, and 33 NC (22 males/11 females) with a mean age of 27.30 \pm 7.84 years. There was no difference in age and sex distribution between the two groups ( $p=0.588$ and $p=$ 0.415 , respectively). Demographic features of patients are summarized in Table 1.

As illustrated in Table 2, compared to NC, the TOS $(p=0.002)$ and OSI values $(p<0.001)$ were higher, while the TAS level $(p<0.001)$ was lower, in patients with AA.

Table 2. Hospital Anxiety and Depression Scale scores and oxidative stress markers in patients with alopecia areata and controls

\begin{tabular}{|c|c|c|c|}
\hline Parameter & $\begin{array}{l}\text { Patients } \\
(n=33)\end{array}$ & $\begin{array}{r}\text { Controls } \\
(n=33)\end{array}$ & $P$-value \\
\hline HADS-A score & $9.45 \pm 3.40$ & $6.18 \pm 3.30$ & $<0.001$ \\
\hline HADS-D score & $8.67 \pm 3.11$ & $4.73 \pm 2.76$ & $<0.001$ \\
\hline TAS [ $\mu$ mol Trolox Eq/l] & $1.24 \pm 0.33$ & $1.58 \pm 0.42$ & $<0.001$ \\
\hline TOS [ $\mu \mathrm{mol} \mathrm{H} 2 \mathrm{O} 2 \mathrm{Eq} / \mathrm{l}]$ & $19.64 \pm 4.54$ & $15.55 \pm 5.46$ & 0.002 \\
\hline OSI [arbitrary units] & $1.69 \pm 0.55$ & $1.05 \pm 0.46$ & $<0.001$ \\
\hline
\end{tabular}


Table 3. Effects of anxiety and depressive scores on oxidative stress markers in patients with alopecia areata

\begin{tabular}{|c|c|c|c|c|c|c|}
\hline Parameter & $\begin{array}{l}\text { Patients with } \\
\text { anxiety } \\
\text { HADS-A > } 10 \\
(n=12)\end{array}$ & $\begin{array}{l}\text { Patients without } \\
\text { anxiety } \\
\text { HADS-A } \leq 10 \\
(n=21)\end{array}$ & $P$-value & $\begin{array}{l}\text { Patients with } \\
\text { depression } \\
\text { HADS-D > } 7 \\
(n=19)\end{array}$ & $\begin{array}{c}\text { Patients without } \\
\text { depression } \\
\text { HADS-D } \leq 7 \\
(n=14)\end{array}$ & $P$-value \\
\hline TAS [ $\mu \mathrm{mol}$ Trolox Eq/l] & $1.32(0.71-1.98)$ & $1.14(0.74-1.86)$ & 0.359 & $1.15(0.71-1.98)$ & $1.27(0.74-1.86)$ & 0.913 \\
\hline TOS $[\mu \mathrm{mol} \mathrm{H} 2 \mathrm{O} 2 \mathrm{Eq} / \mathrm{l}]$ & $21.13(12.5-25.25)$ & $20.75(12.25-29.25)$ & 0.627 & $21.88(12.5-29.25)$ & $20.32(12.25-23.13)$ & 0.126 \\
\hline OSI [arbitrary units] & $1.59(0.64-2.57)$ & $1.6(0.94-2.72)$ & 0.681 & $1.77(0.64-2.71)$ & $1.51(0.98-2.72)$ & 0.362 \\
\hline
\end{tabular}

TAS - total antioxidant status, TOS - total oxidant status, OSI - oxidative stress index.

Table 4. Correlation between Hospital Anxiety and Depression Scale scores and oxidative stress markers in patients with alopecia areata

\begin{tabular}{lcccc}
\hline Parameter & \multicolumn{2}{c}{ HADS-A score } & \multicolumn{2}{c}{ HADS-D score } \\
\cline { 2 - 5 } & $r$ & $p$ & $r$ & $p$ \\
\hline TAS & 0.178 & 0.321 & 0.132 & 0.464 \\
\hline TOS & 0.071 & 0.693 & 0.189 & 0.293 \\
\hline OSI & -0.148 & 0.412 & 0.021 & 0.909 \\
\hline
\end{tabular}

HADS - Hospital Anxiety and Depression Scale, HADS-A - HADS-Anxiety, HADS-D - HADS-Depression, TAS - total antioxidant status, TOS - total oxidant status, OSI - oxidative stress index.

According to the cut-off values, anxiety symptoms (HADS-A > 10) were present in 12 (36.4\%) patients and 5 (15.2\%) NC, while depression symptoms (HADS-D > 7) were present in 19 (57.6\%) patients and 6 (18.2\%) NC. HADS-A ( $9.45 \pm 3.40$ vs. $6.18 \pm 3.30 ; p<0.001)$ and HADS-D (8.67 \pm 3.11 vs. $4.73 \pm 2.76 ; p<0.001)$ scores were higher in AA patients compared to NC (Table 2 ).

There was no significant difference in TAS, TOS, or OSI levels between patients with high anxiety scores (HADS-A $>10)$ and low anxiety scores (HADS-A $\leq 10$ ). Similarly, patients with high (HADS-D > 7) and low (HADS-D $\leq 7$ ) depression scores did not differ in terms of TAS, TOS, or OSI values (Table 3).

Patients' HADS-A and HADS-D scores did not correlate with TAS, TOS, or OSI values $(p>0.05)$ (Table 4).

\section{Discussion}

The skin is the target of OXS due to ROS produced by exposure to physical and chemical agents [4]. Researchers have shown that OXS plays an important role in skin aging [17], as well as various skin diseases such as psoriasis [18], lichen planus [19], and AA [6, 7].

Oxidative stress, which is an imbalance between ROS production and detoxification, may cause cellular damage by interacting with macromolecules such as protein, lipid, and DNA. Levels of ROS in the organism are maintained in a normal range through antioxidant agents such as superoxide dismutase (SOD), glutathione peroxidase (GPx), glutathione, and vitamins $C$ and $E[5]$.
To date, many studies have been conducted to assess the OXS status of patients with AA, and contradictory results have been obtained. Abdel Fattah et al. [20] and Naziroglu et al. [21] found higher levels of lipid peroxidation products and lower levels of antioxidant enzyme activities in patients with AA than in NC. Similarly, Yenin et al. [7] reported high levels of malondialdehyde, a lipid peroxidation product, and low GPx and SOD activities in AA patients compared to NC, indicating that AA patients are in a state of enhanced OXS. In our study, TOS and OSI values were higher, and the TAS level was lower, in AA patients compared to NC, as reported previously [6, 22].

Contrary to the above observations, Akar et al. [23] observed that SOD and GPx activities in the scalps of AA patients were significantly higher than in the NC. The authors also found that SOD and GPx activities were increased in the early stages of the disease compared to the late stage. Another study revealed higher levels of SOD and GPx in patients with AA than in NC. In the same study, no significant increase in plasma malondialdehyde level was observed in the patient group [24]. Interestingly, Motor et al. [25] reported that TAS, TOS, and OSI values were similar in the AA patients and NC. The discrepancy in the results of these studies may be due to differences in the sample size, disease duration, and extent of hair loss, as well as the use of different samples in each study. Collectively, these results suggest that increased OXS and impaired antioxidant capacity may be related to the pathogenesis of $A A$.

Since AA can seriously affect individuals' self-esteem and cosmetic appearance, these patients may be prone to psychiatric disorders such as depression, anxiety, and social phobia [8]. Another approach that explains the relationship between $A A$ and psychological disorders is that stressful life events may play an important role in the onset and/or exacerbation of the disease $[9,10]$.

Sellami et al. [11] reported that $38 \%$ of patients with AA had depression and $62 \%$ had anxiety symptoms. In addition, many studies have shown high rates of depression and anxiety in AA patients compared to the general population $[12,26]$. Consistent with these studies, we found that depression and anxiety scores were higher in patients with AA compared to NC, which indicates a relationship between $A A$ and psychological disorders. 
Moreover, some studies indicated that antidepressants may have beneficial effects on AA in AA sufferers with psychiatric disorders [27, 28].

On the other hand, Güles et al. [29] reported that the scores for stressful life events, anxiety, and depression were not significantly different between AA patients and NC. These contradictory results can be attributed to differences in the scales used to screen for psychiatric disorders, and to differences in population size among these studies.

The brain, which shows high oxygen utilization and has a lipid-rich environment, is susceptible to the harmful effects of ROS [30]. Studies have shown that OXS may play a role in the pathophysiology of various psychiatric diseases, including anxiety and depressive disorders [31, 32]. To our knowledge, no study has investigated the effects of psychiatric disorders on OXS in AA patients. In the current study, we evaluated the relationship between HADS subscale scores and OXS markers in patients with AA. Our findings showed that there was no difference in terms of TAS, TOS, or OSI values between patients with and without anxiety or between patients with and without depression. Moreover, HADS scores did not correlate with TAS, TOS, or OSI values in patients with AA. Similarly, Karababa et al. [33], using the HADS scale, did not find an association between OXS and psychological status in psoriasis patients. In another study, the relationship between OXS and HADS scores was investigated in acne patients, and it was found that higher HADS subscale scores did not lead to a further increase in OXS [34]. In our opinion, these results may be attributed to the use of the HADS rather than clinical psychiatric evaluations of AA patients, and to the small numbers of cases included.

The main limitation of this study was the small number of AA cases. Another limitation was the use of the HADS rather than clinical psychiatric assessments for determining the anxiety and depression status of AA patients.

\section{Conclusions}

Consequently, both the HADS subscale scores and OXS were higher in AA patients compared to NC. However, OXS was unrelated to the anxiety or depression scores of the AA patients. In addition, in patients with AA who are at risk of depression or anxiety, psychological intervention may be part of the treatment. To better understand the effect of psychiatric disorders on OXS in AA patients, further studies should be performed with large patient populations.

\section{Conflict of interest}

The authors declare no conflict of interest.

\section{References}

1. Gilhar A, Etzioni A, Paus R. Alopecia areata. N Engl J Med 2012; 366: 1515-25.
2. Alkhalifah A, Alsantali A, Wang E, et al. Alopecia areata update: part I. Clinical picture, histopathology, and pathogenesis. J Am Acad Dermatol 2010; 62: 177-88.

3. Wang E, McElwee KJ. Etiopathogenesis of alopecia areata: why do our patients get it? Dermatol Ther 2011; 24: 337-47.

4. Bickers DR, Athar M. Oxidative stress in the pathogenesis of skin disease. J Invest Dermatol 2006; 126: 2565-75.

5. Özcan O, Erdal H, Çakırca G, Yönden Z. Oxidative stress and its impacts on intracellular lipids, proteins and DNA. J Clin Exp Invest 2015; 6: 331-6.

6. Bilgili SG, Ozkol H, Karadag AS, et al. Serum paraoxonase activity and oxidative status in subjects with alopecia areata. Cutan Ocul Toxicol 2013; 32: 290-3.

7. Yenin JZ, Serarslan G, Yonden Z, Ulutas KT. Investigation of oxidative stress in patients with alopecia areata and its relationship with disease severity, duration, recurrence and pattern. Clin Exp Dermatol 2015; 40: 617-21.

8. Hunt N, McHale S. The psychological impact of alopecia. BMJ 2005; 331: 951-3.

9. Kakourou T, Karachristou K, Chrousos G. A case series of alopecia areata in children: impact of personal and family history of stress and autoimmunity. J Eur Acad Dermatol Venereol 2007; 21: 356-9.

10. Manolache L, Benea V. Stress in patients with alopecia areata and vitiligo. J Eur Acad Dermatol Venereol 2007; 21: 921-8.

11. Sellami R, Masmoudi J, Ouali U, et al. The relationship between alopecia areata and alexithymia, anxiety and depression: a case-control study. Indian J Dermatol 2014; 59: 421.

12. Chu SY, Chen YJ, Tseng WC, et al. Psychiatric comorbidities in patients with alopecia areata in Taiwan: a case-control study. Br J Dermatol 2012; 166: 525-31.

13. Erel O. A novel automated method to measure total antioxidant response against potent free radical reactions. Clin Biochem 2004; 37: 112-9.

14. Erel O. A new automated colorimetric method for measuring total oxidant capacity. Clin Biochem 2005; 38: 1103-11.

15. Bjelland I, Dahl AA, Haug TT, Neckelmann D. The validity of the Hospital Anxiety and Depression Scale. An updated literature review. J Psychosom Res 2002; 52: 69-77.

16. Aydemir Ö, Güvenir T, Küey L, Kültür S. Validity and reliability of Turkish version of hospital anxiety and depression scale. Turk J Psychiatry 1997; 8: 280-7.

17. Rinnerthaler M, Bischof J, Streubel MK, et al. Oxidative stress in aging human skin. Biomolecules 2015; 5: 545-89.

18. Gabr SA, Al-Ghadir AH. Role of cellular oxidative stress and cytochrome $\mathrm{c}$ in the pathogenesis of psoriasis. Arch Dermatol Res 2012; 304: 451-7.

19. Aly DG, Shahin RS. Oxidative stress in lichen planus. Acta Dermatovenerol Alp Pannonica Adriat 2010; 19: 3-11.

20. Abdel Fattah NSA, Ebrahim AA, El Okda ES. Lipid peroxidation/antioxidant activity in patients with alopecia areata. J Eur Acad Dermatol Venereol 2011; 25: 403-8.

21. Naziroglu M, Kokcam I. Antioxidants and lipid peroxidation status in the blood of patients with alopecia. Cell Biochem Funct 2000; 18: 169-73.

22. Bakry OA, Elshazly RM, Shoeib MA, Gooda A. Oxidative stress in alopecia areata: a case-control study. Am J Clin Dermatol 2014; 15: 57-64.

23. Akar A, Arca E, Erbil H, et al. Antioxidant enzymes and lipid peroxidation in the scalp of patients with alopecia areata. J Dematol Sci 2002; 29: 85-90.

24. Güngör Ş, Akbay G, Öğüş E, et al. Changes of lipid peroxidation and antioxidant system in serum and tissues of pa- 
tients with alopecia areata. Turkiye Klin J Dermatol 2008; 18: 141-5.

25. Motor S, Ozturk S, Ozcan O, et al. Evaluation of total antioxidant status, total oxidant status and oxidative stres index in patients with alopecia areata. Int J Clin Exp Med 2014; 7: 1089-93.

26. Aghaei S, Saki N, Daneshmand E, Kardeh B. Prevalence of psychological disorders in patients with alopecia areata in comparison with normal subjects. ISRN Dermatol 2014; 2014: 304370.

27. Abedini H, Farshi S, Mirabzadeh A, Keshavarz S. Antidepressant effects of citalopram on treatment of alopecia areata in patients with major depressive disorder. J Dermatolog Treat 2014; 25: 153-5.

28. Cipriani R, Perini GI, Rampinelli S. Paroxetine in alopecia areata. Int J Dermatol 2001; 40: 600-1.

29. Güleç AT, Tanriverdi N, Dürü C, et al. The role of psychological factors in alopecia areata and the impact of the disease on the quality of life. Int J Dermatol 2004; 43: 352-6.

30. Ng F, Berk M, Dean O, Bush Al. Oxidative stress in psychiatric disorders: evidence base and therapeutic implications. Int J Neuropsychopharmacol 2008; 11: 851-76.

31. Bajpai A, Verma AK, Srivastava M, Srivastava R. Oxidative stress and major depression. J Clin Diagn Res 2014; 8: CCO4-7.

32. Guney E, Fatih Ceylan M, Tektas A, et al. Oxidative stress in children and adolescents with anxiety disorders. J Affect Disord 2014; 156: 62-6.

33. Karababa F, Yesilova Y, Turan E, et al. Impact of depressive symptoms on oxidative stress in patients with psoriasis. Redox Rep 2013; 18: 51-5.

34. Awad SM, Morsy H, Sayed AA, et al. Oxidative stress and psychiatric morbidity in patients with facial acne. J Cosmet Dermatol 2018; 17: 203-8. 